\title{
Testosterone Replacement Therapy in Men with Untreated or Treated Prostate Cancer: Do We Have Enough Evidences?
}

\author{
Myong Kim ${ }^{1}$ (i), Seok-Soo Byun ${ }^{2}$ (i) , Sung Kyu Hong ${ }^{2}$ (iD \\ ${ }^{1}$ Department of Urology, Ewha Womans University Seoul Hospital, Seoul, ${ }^{2}$ Department of Urology, Seoul National University Bundang \\ Hospital, Seongnam, Korea
}

\begin{abstract}
Purpose: To investigate the oncologic safety of testosterone replacement therapy (TRT) in men with untreated or treated prostate cancer.

Materials and Methods: We systematically searched PubMed, Embase, and Cochrane library database from January 1941 to March 2019.

Results: In total, 36 articles met the eligibility criteria for this systematic review. They included a total of 2,459 TRT-treated patients, with a median of 20 patients per study (range: 1-1,142). Except for four studies, all were single-armed studies with poor quality scores (median MINOR, 9 of 24). Of the 36 studies, prostate cancer was managed through active surveillance (AS), in 5 studies; radical prostatectomy, in 11 studies; radiation therapy, in 5 studies; multiple intervention modalities, in 5 studies; and systemic therapy, in 9 studies. In comparison with TRT-treated and untreated patients, the pooled risk ratio (RR) was not significantly higher than one in comparisons of risk for disease progression (pooled RR, 0.83; 95\% confidence interval, 0.57-1.21). The results of systematic review implied that TRT might be harmful in men with advanced disease (progression rate: $38.5 \%-100.0 \%)$, who undergo AS (15.4\%-57.1\%), and who successfully treated but having high-risk disease $(0.0 \%-50.0 \%)$.

Conclusions: Compared to TRT-untreated patients, TRT-treated patients may not have increased risks for disease progression in prostate cancer. However, the quality of currently available evidence is extremely poor. TRT may be harmful in men with advanced disease burden, in those with untreated prostate cancer undergoing AS, and in those with successfully treated prostate cancer but having high-risk disease.
\end{abstract}

Keywords: Eunuchism; Hormone replacement therapy; Prognosis; Prostatic neoplasms; Safety; Testosterone

This is an Open Access article distributed under the terms of the Creative Commons Attribution Non-Commercial License (http://creativecommons.org/licenses/by-nc/4.0) which permits unrestricted non-commercial use, distribution, and reproduction in any medium, provided the original work is properly cited.

\section{INTRODUCTION}

Prostate-specific antigen (PSA) screening has resulted in a robust migration of the clinical stage in newly detected prostate cancers [1,2]. The American Cancer
Society predicted 180,890 new prostate cancer cases in 2016, with $35 \%-40 \%$ of those being low risk [3]. This increasing detection of low-risk disease enables active surveillance as a viable treatment option for prostate cancer [4-6]. Moreover, owing to the increased life ex-

Received: Feb 11, 2020 Revised: Apr 9, 2020 Accepted: Apr 13, 2020 Published online Jun 25, 2020

Correspondence to: Sung Kyu Hong (iD https://orcid.org/0000-0002-8344-6774

Department of Urology, Seoul National University Bundang Hospital, 82 Gumi-ro 173beon-gil, Bundang-gu, Seongnam 13620, Korea.

Tel: : +82-31-787-7343, Fax: +82-31-787-4057, E-mail: skhong@snubh.org 
pectancy caused by downward stage migration, an expanding population of patients successfully treated for prostate cancer strongly desire testosterone replacement therapy (TRT) for hypogonadal symptoms accompanied by decreased serum testosterone levels [7].

However, the effectiveness of TRT for men with untreated or treated prostate cancer is controversial. Per the demonstration of hormonal responsiveness in 1941 by Huggins and Hodges [8], the relationship between serum testosterone and prostatic health has been thought to be an "old dogma" in the form of "fuel for a fire." The United States Food and Drug Administration (FDA) stated, in all testosterone package inserts, that TRT is contraindicated in men with known or suspected prostate cancer, but it did not substantiate this contraindication [9]. The clinical guidelines by Endocrine Society recommend against treating hypogonadism in men with prostate cancer, citing lack of sufficient data to make a general recommendation in men previously treated for prostate cancer ('recommendation with low quality evidence') [10].

Meanwhile, the European Association of Urology stated that there is no conclusive evidence that TRT increases the risk of prostate cancer ('level of evidence $=4^{\prime}$ ), and that men with prostate cancer can receive TRT with careful monitoring for prostate safety ('level of evidence=3') [11]. Similarly, the recent treatment guideline by the American Urologic Association stated that patients should be informed that there is inadequate evidence for TRT ('expert opinion'), but TRT can be considered in men who have undergone radical prostatectomy with favorable pathology (e.g., negative margins, negative seminal vesicles, negative lymph nodes), without PSA recurrence [12].

The prescribing patterns of TRT in patients with treated [13] and untreated prostate cancer [14] are rapidly changing. In the Unites States, 94\% of urologists prescribed TRT to patients who had been treated for prostate cancer previously [13]. Additionally, 65\% of Canadian urologists stated that they would offer TRT to men who were on active surveillance for prostate cancer [14]. However, it remains unclear whether there are sufficient evidences for these beliefs. To our knowledge, no randomized studies have been reported regarding the safety of TRT in men with untreated or treated prostate cancer. There have been some review articles ('mainly by authors advocating TRT in prostate cancer') summarizing optimistic results from small- scale studies for TRT in patients with prostate cancer who have undergone active surveillance or other treatments [15-21]. However, the existing evidences have rarely been evaluated and synthesized in a systematic manner. Owing to the scarcity of reports in this regard, quality assessment and summation of these existing evidences ('if possible') to reach a reasonable conclusion are necessary. Therefore, we performed a systematic review and meta-analysis of published literature investigating the safety of TRT in men with untreated and treated prostate cancer.

\section{MATERIALS AND METHODS}

\section{Search strategy for relevant studies}

The entire process of this systematic review and meta-analysis followed the recent MOOSE and PRISMA recommendations [22,23]. We systematically searched online PubMed, Embase, and Cochrane library database from their respective inspections until March 2019. Our overall search strategies included terms for prostate cancer (prostatic neoplasm, prostate carcinoma, or prostate adenocarcinoma), treatments (watchful waiting, active surveillance, focal therapy, surgery, radiation therapy, androgen deprivation therapy, or chemotherapy), testosterone deficiency (hypogonadism or androgen deficiency), and hormone replacement therapy (testosterone replacement and testosterone supplementation). Detailed queries for the search strategy are presented in Appendix. Some studies were manually searched by referring the review articles or original research articles on similar subjects.

\section{Selection criteria of eligible studies for meta-analysis}

Original research articles or abstracts, articles in which the subjects were only patients with prostate cancer with or without treatment; those in which patients received TRT owing to symptomatic testosterone deficiency; those in which oncological outcome parameters were objectively described using standard investigation tools, such as biochemical recurrence or radiographic progression; those in which the sample size was provided; and double-armed studies (TRT-treated vs. TRT-untreated) in which the risks for progression in each group were presented separately for the estimation of risk ratio (RR) were included in this systematic review. In case of suspected duplication of patient 


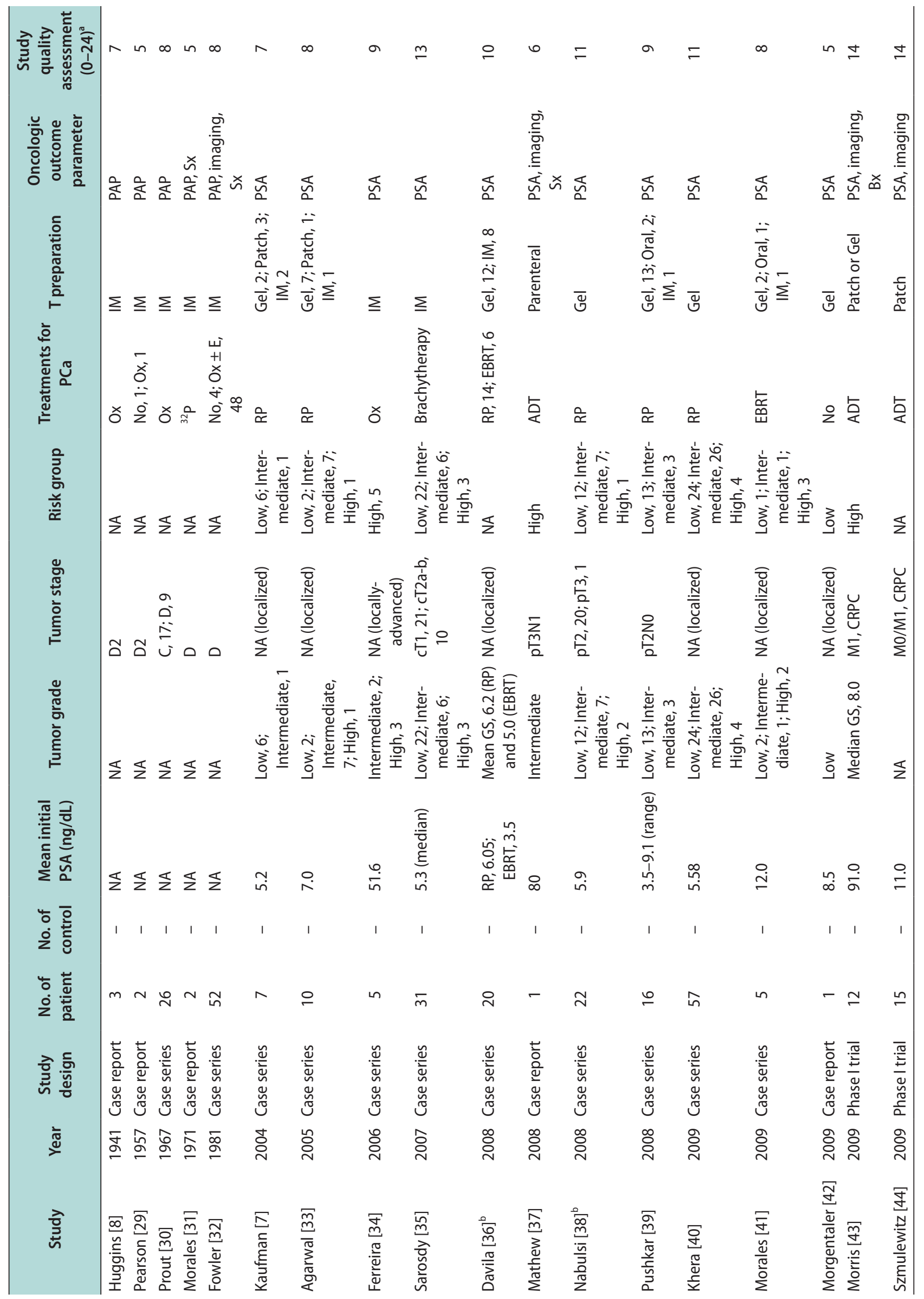




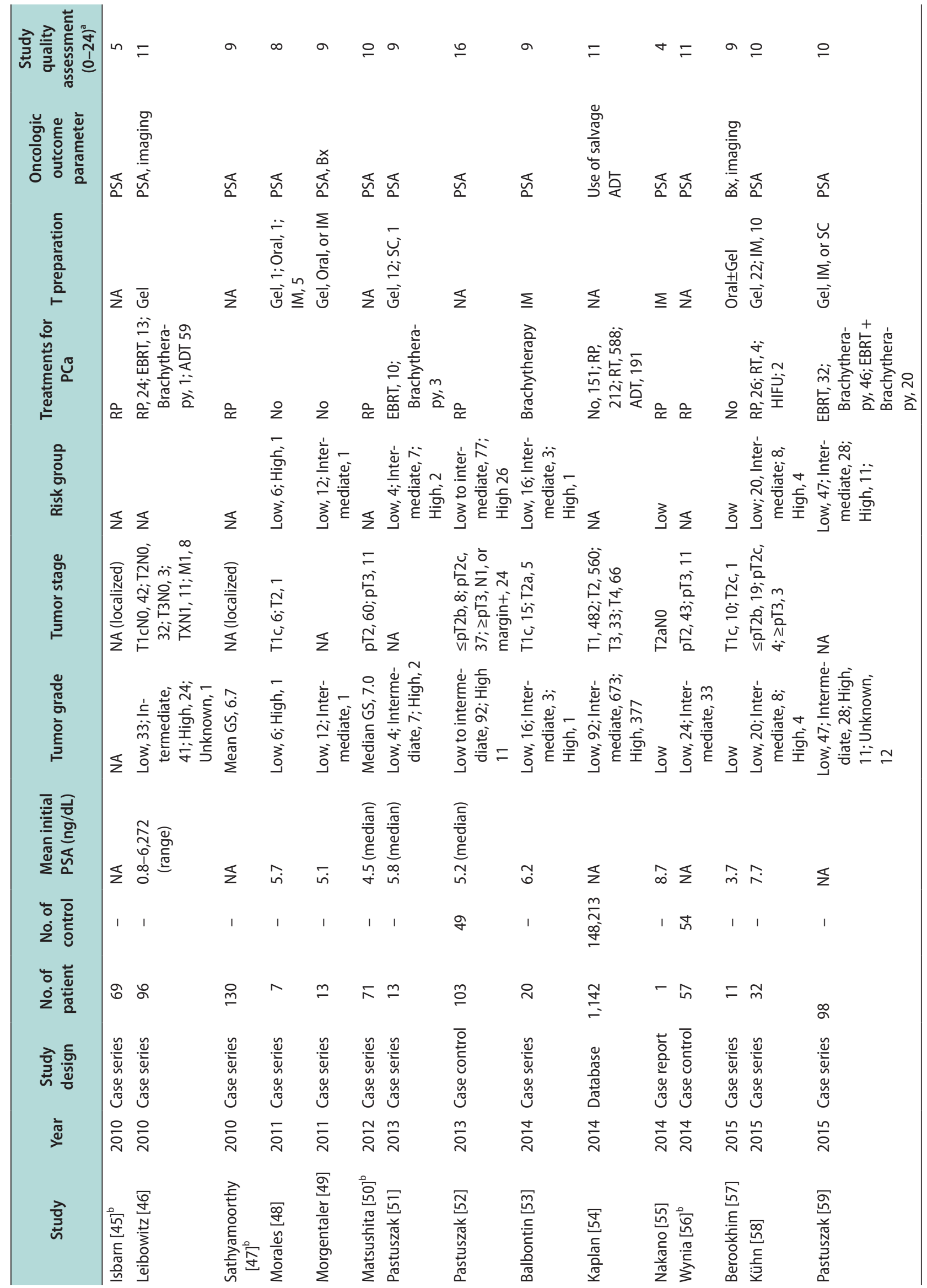


data, the most recently published or the most informative single article was selected. If the study population underwent two or more treatment modalities, each datum was processed separately by the treatment type, or presented all at once when the separation was impossible. Owing to the scarcity of double-armed studies, all single-armed studies and case reports were included in the eligible studies. Studies that failed to satisfy the previously mentioned inclusion criteria, review articles or letters, laboratory studies, such as studies on ex-vivo or animal models, and studies with insufficient data to estimate the effects of TRT on oncological outcomes and its RR for progression were excluded.

To minimize bias, abstract screening and full text assessment for eligibility were independently performed by all three reviewers (MK, SSB, and SKH). All screened abstracts were classified into three categories: not eligible, unclear, and potentially eligible. The full texts of "potentially eligible" and "unclear" studies were obtained and assessed for eligibility. Any disagreements between the three reviewers were resolved by consensus.

\section{Data extraction and quality assessments}

The extracted data elements were 1) overall characteristics of the eligible studies: name of the first author, publication year, study design, population size (intervention and control group); 2) characteristics of the patients: mean PSA level at initial diagnosis, tumor grade, stage, and risk group; 3) treatment data: type of treatment for prostate cancer, type of TRT; and 4) outcome parameters: oncologic parameters for progression, median follow-up periods from initial diagnosis and commencement of TRT, and the number and risks for progression in each arm. The study quality was assessed independently by all three reviewers using the MINOR criteria (score range: 0-24) [24]. Any disagreement was resolved by discussion.

\section{Statistical analysis}

\section{1) Primary analysis (narrative systematic review)}

Existing evidences for TRT in men with untreated or treated prostate cancer were summarized (Table 1). The effects of TRT on prostate cancer progression were evaluated according to the treatment modality, as follows: active surveillance, radical prostatectomy, radiation therapy, multiple modalities, or systemic therapy 


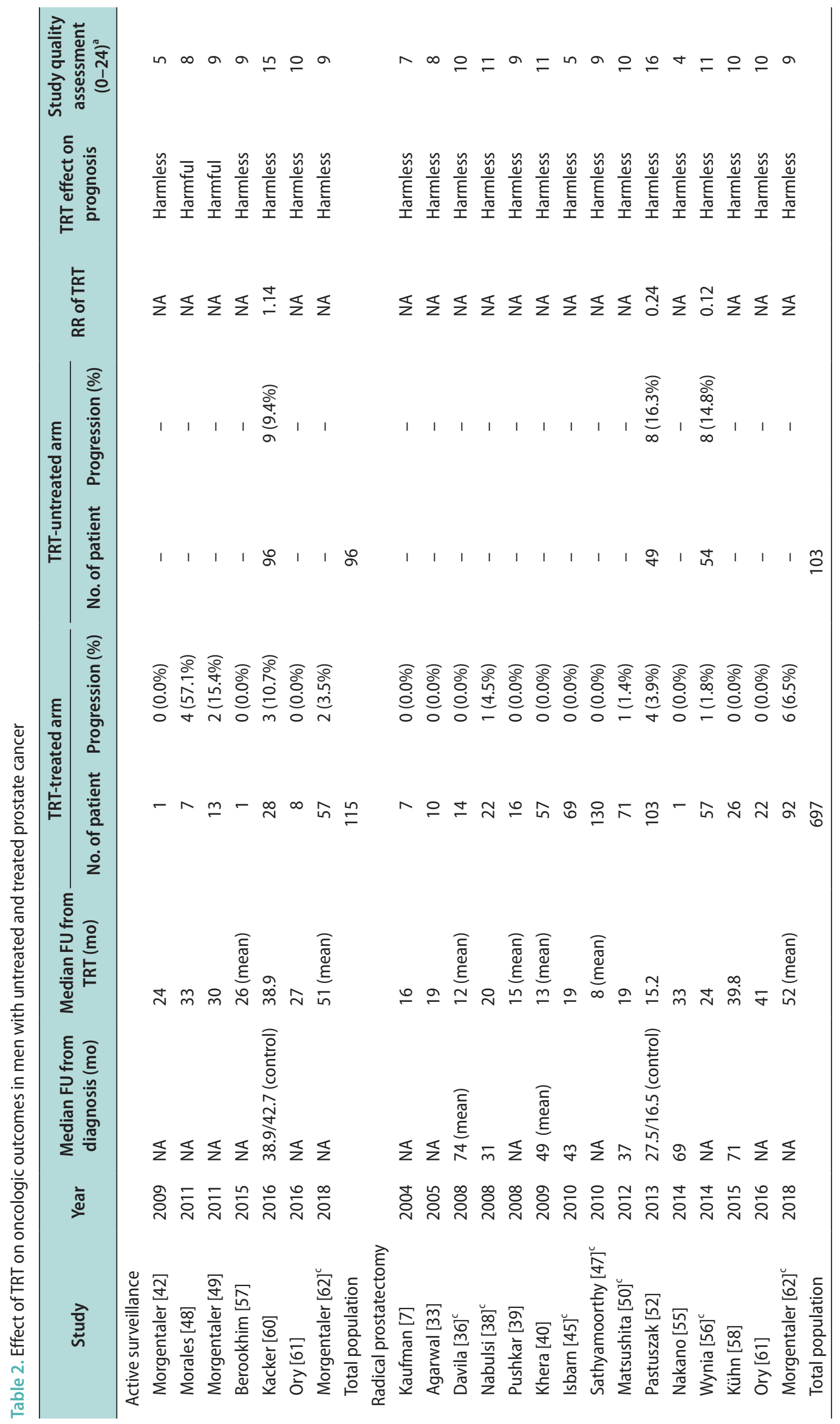




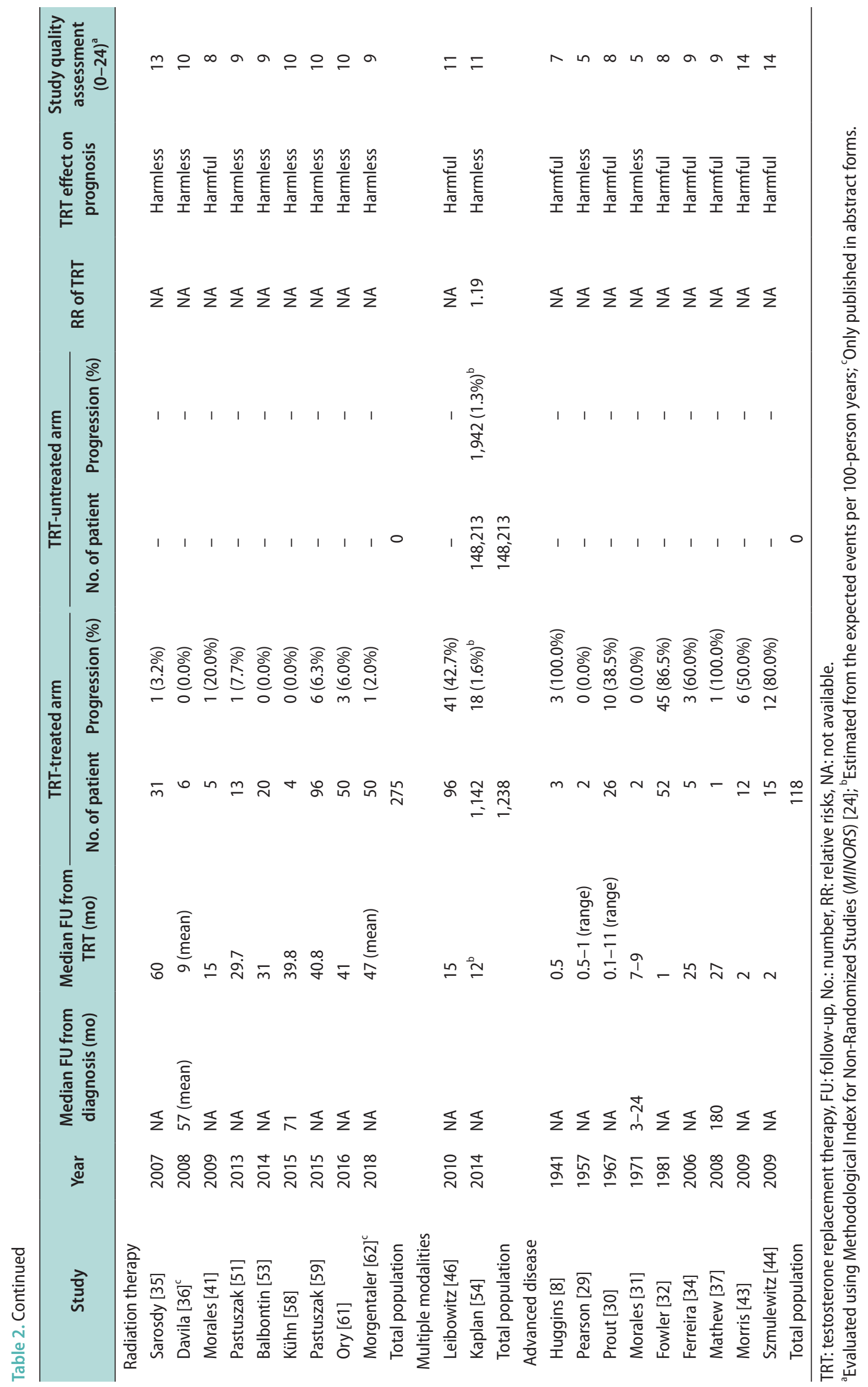




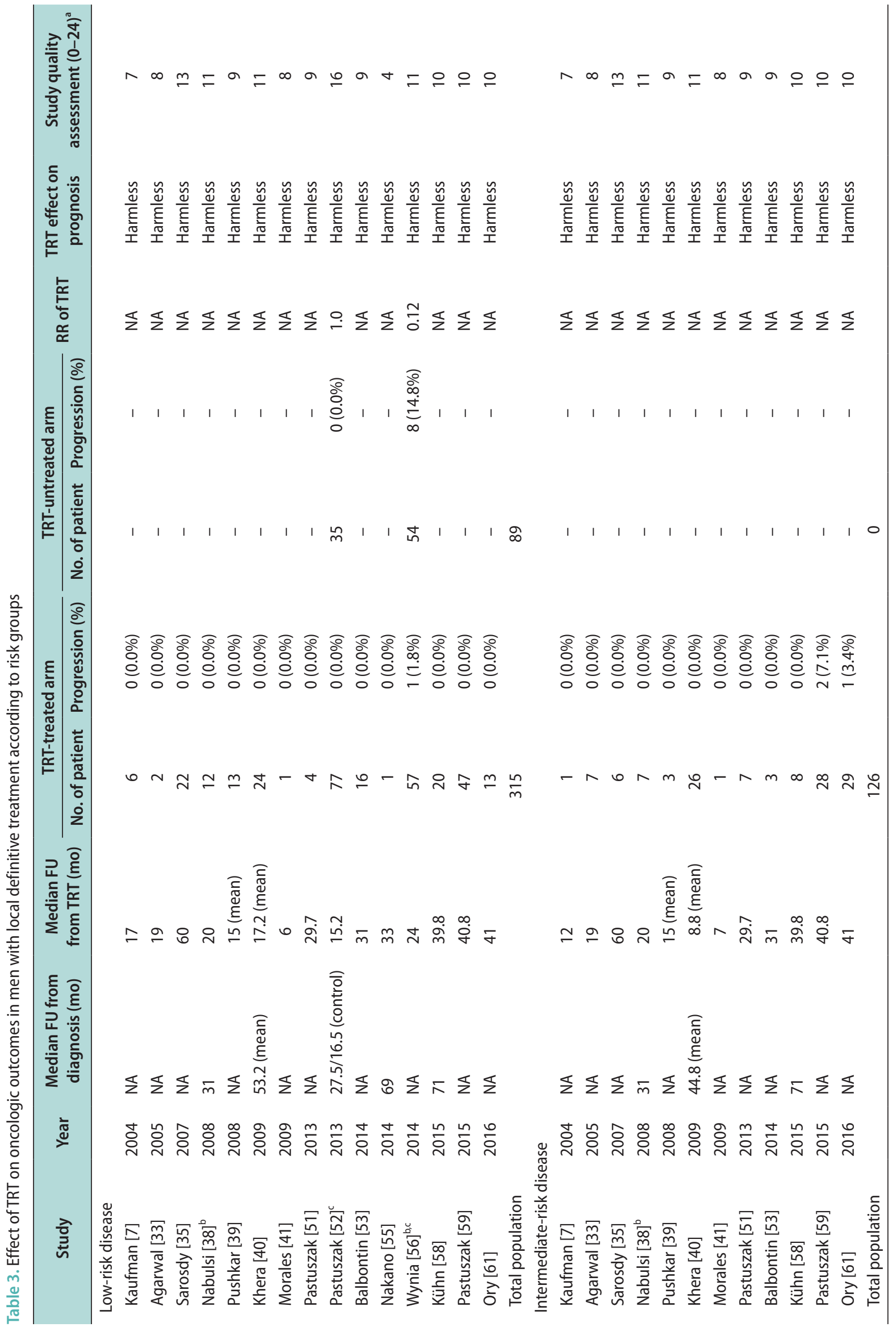




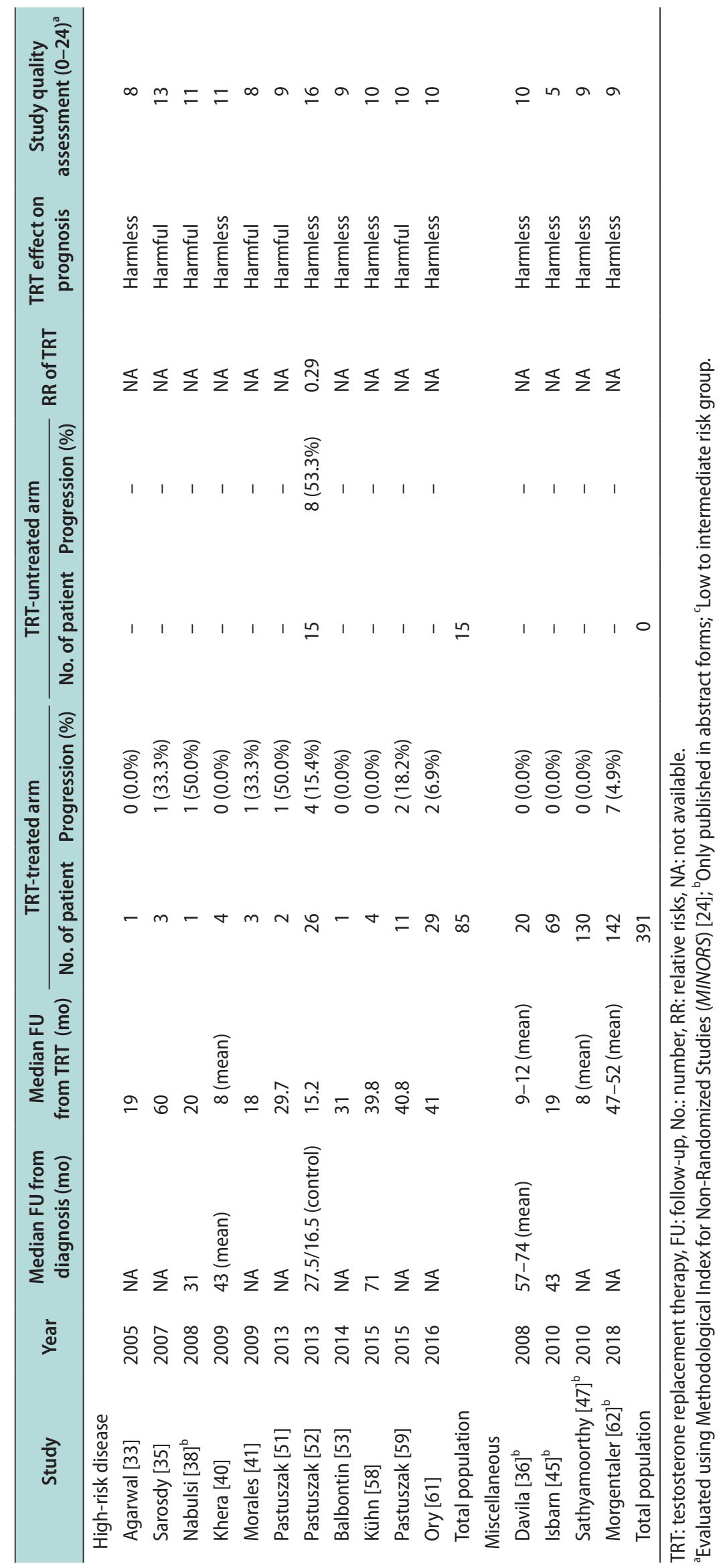


for advanced disease (Table 2). The effects of TRT on the prognosis of the patients in each study were assessed and the qualities of evidences were evaluated. In studies with local-intent modalities such as radical prostatectomy or radiation therapy, the effects of TRT on oncologic outcomes were assessed according to the risk groups (low, intermediate, and high; Table 3).

\section{2) Secondary analysis (meta-analysis)}

Using the double-armed studies, quantitative synthesis was performed to assess the risk of progression of prostate cancer in patients receiving TRT (Fig. 1). As a summarizing statistic for meta-analysis, the pooled RR was utilized. Owing to the relatively small number of selected studies, a fixed-effects model was adopted for meta-analysis. Weights between the studies were estimated using the Mantel-Haenszel method to obtain the summary with a pooled RR and its $95 \%$ confidence interval (CI) [25]. A pooled RR $>1$ indicated increased risks for disease progression in the study group (TRTtreated group) relative to the reference group (TRTuntreated group), and would be considered statistically significant if the $95 \%$ CI did not overlap the pooled $\mathrm{MD}$ value of one, with $\mathrm{p}<0.05$. Inter-study heterogeneity was assessed using the Higgin's H-test ( $\mathrm{I}^{2}$ statistic) [26] and heterogeneity $x^{2}$ test [27], and $p>0.05$ indicated the absence of significant heterogeneity. Possibilities of publication bias were assessed by drawing a funnel plot [28]. A non-commercialized software (RevMan version 5.3.5; The Nordic Cochrane Center, The Cochrane Collaboration, Copenhagen, Denmark) was used for data synthesis.

\section{Ethics statement}

The present study protocol waived the requirement for approval by the institutional review board, because we reviewed and analyzed already published articles.

\section{RESULTS}

A methodological flow chart of the entire systematic review process is shown in Fig. 2. Our search strategy identified 208 articles (PubMed, 78 articles; Embase, 124 articles; Cochrane library database, 6 articles). Additionally, 29 articles were found by manual searching. After removal of duplicates, 205 abstracts were independently screened by three independent reviewers. After abstract screening, 125 articles were included for full text assessment. After careful review of the full articles, 89 articles were excluded for the following reasons: 37 were review articles, 12 were letters to the editor, 12 were out of scope, 15 covered the relevant subject but failed to satisfy the inclusion criteria in detailed methodology, 2 lacked eligibility data, and 11 studies were excluded owing to duplication of population. Eventually, 36 studies were selected for the narrative systematic review [7,8,29-62]. Among them, four studies had a double-armed design [52,54,56,60]; quantitative synthesis was performed using these studies to assess the risk of prostate cancer progression in patients receiving TRT.

\section{Characteristics of included studies}

The characteristics of the eligible studies are shown in Table 1. The 36 eligible studies included 2,459 TRTtreated patients, with a median number of 20 TRTtreated patients per study (range: 1-1,142). None of the selected studies were randomized prospective studies. Of the 36 included studies, two were single-armed prospective studies [43,44], four were double-armed retrospective studies [52,54,56,60], and the remaining studies had single-armed retrospective features (case

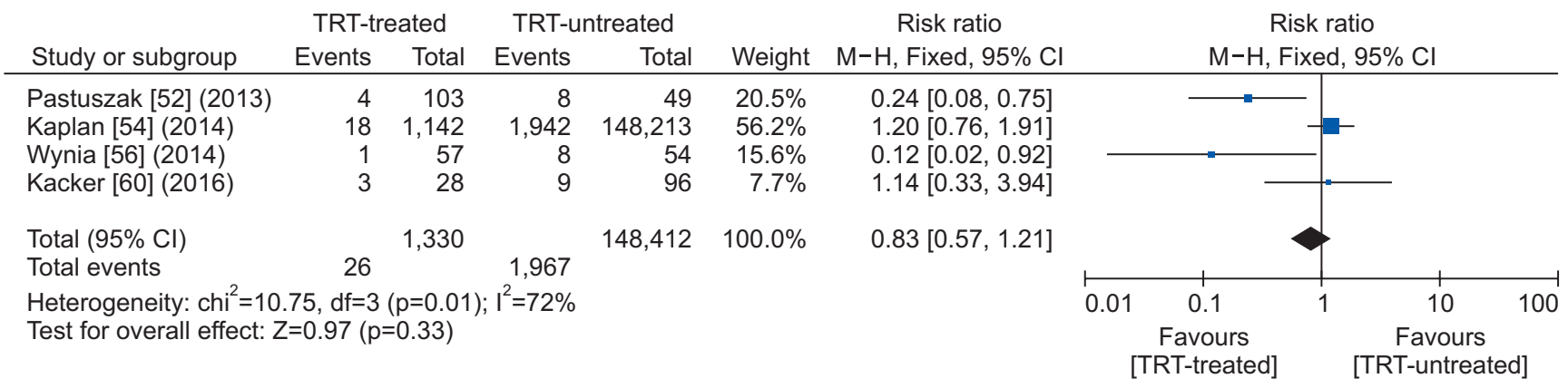

Fig. 1. A forest plot assessing the risk ratio for prostate cancer progression in patients with or without testosterone replacement therapy (TRT), using the fixed-effects model. M-H: Mantel-Haenszel, Cl: confidence interval, df: degree of freedom. 


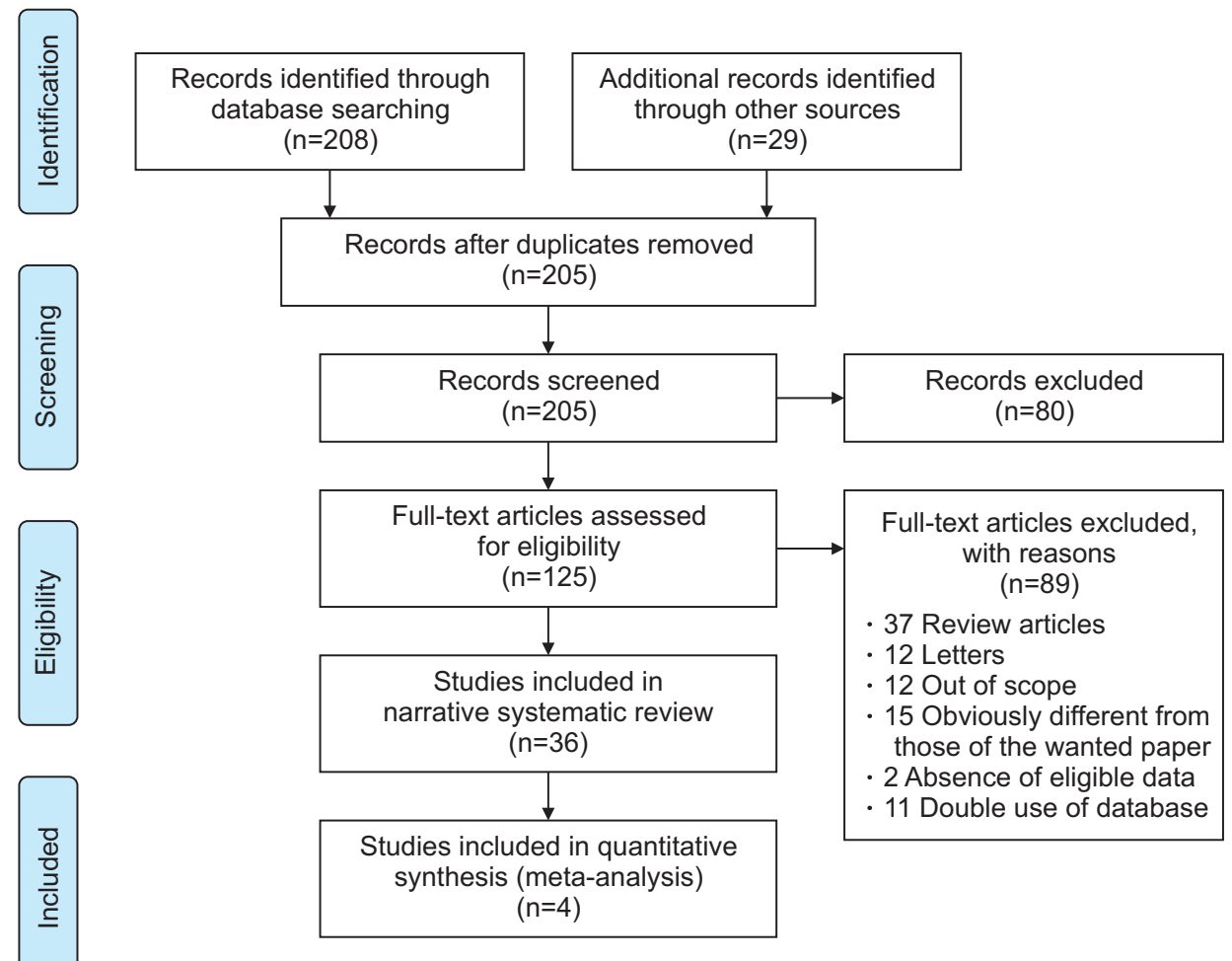

Fig. 2. Methodological flow chart of the systematic review.

series or case reports) [7,8,29-42,45-51,53,55,57-59,61,62]. Patients underwent active surveillance in 5 of the 36 studies [42,48,49,57,60], radical prostatectomy in 11 studies $[7,33,38-40,45,47,50,52,55,56]$, radiation therapy in 5 studies [35,41,51,53,59], multiple intervention modalities in 6 studies [36,46,54,58,61,62], and systemic therapy in 9 studies [8,29-32,34,37,43,44], for the management of underlying prostate cancer. Except for some old studies conducted in the pre-PSA era [8,29-32], a database study [54], and a study with active surveillance [57], almost all studies utilized PSA as an oncologic outcome parameter [7,33-53,55,56,58-62]. As almost all included studies had single-armed retrospective feature, the quality scores measured by the MINOR criteria [24] were extremely low (median, 9 [range: 4-16]; Table 1). There was no significant correlation between population size and quality scores ( $\mathrm{p}=0.330$, by Pearson's correlation analysis).

\section{Evidences for effects of testosterone replacement therapy in patients with active} surveillance

Existing evidences for TRT in patients with active surveillance comprised 6 studies, which including a total of 115 TRT-treated patients, and 90 TRT-untreated controls (Table 2) $[42,48,49,57,60-62]$. Among the 6 included studies, only one was designed as a doublearmed study [60], and all others were case reports or case series $[42,48,49,57,61,62]$. The median quality score of the six studies was as low as 9 (range: $5-15$ ). Of the six studies, the results of two studies implied that TRT might have harmful effects on the prognosis of patients with active surveillance (progression rate: 15.4\%-57.1\% during 30-33 months of follow-up; Table 2) [48,49].

\section{Evidences for effects of testosterone replacement therapy in patients with radical prostatectomy}

Evidences for effects of TRT in patients with radical prostatectomy comprised 15 studies, which included a total of 697 TRT-treated patients and 103 TRT-untreated controls (Table 2) [7,33,36,38$40,45,47,50,52,55,56,58,61,62]$. All the included 15 studies implied that TRT might be harmless in patients with radical prostatectomy (progression rate: $0.0 \%-6.5 \%$ during 8-52 months of follow-up; Table 2). However, all included studies were case reports or case series, except for two double-armed retrospective studies [52,56], with poor quality scores (median MINOR score, 10 [range: 4-16]; Table 2). 


\section{Evidences for effects of testosterone replacement therapy in patients with radiation therapy}

Evidences for effects of TRT in patients with radiation therapy comprised 9 studies, which included a total of 275 TRT-treated patients (Table 2) [35,36,41,51,53,58,59,61,62]. As all included studies were case series, this study population had no TRT-untreated controls, and the median quality score was as low as 10 (range: 8-13). Of the nine studies, the results of one study implied that TRT might have harmful effects on the prognosis of patients with radiation therapy (progression rate: $20.0 \%$ during 15 months of follow-up; Table 2).

\section{Evidences for effects of testosterone replacement therapy in patients with advanced disease}

Evidences for TRT in patients with advanced disease comprised 9 studies, which included a total of 118 TRT-treated patients (Table 2) [8,29-32,34,37,43,44]. As all included studies were single-armed studies (Four case reports [8,29,31,37], three case series [30,32,34], and two phase I trials [43,44]), this study population had no TRT-untreated controls, and the median quality score was as low as 8 (range: $5-14$ ). Of the nine studies, the results from seven studies implied that TRT might have harmful effects in the prognosis of patients with advanced disease (progression rate: $38.5 \%-100.0 \%$ during 0.1-27.0 months of follow-up; Table 2).

\section{Other evidences for effects of testosterone replacement therapy in patients with prostate cancer}

Data from two studies on the effects of TRT in patients with multiple modalities could not be processed separately according to the type of treatment modality (Table 2) [46,54], because one study failed to present the oncologic outcomes according to the treatment modality [46], and the other was a database study [54]. Underlying prostate cancers were managed with active surveillance, radical prostatectomy, radiation therapy, or systemic therapy in these two studies [46,54]. One case series implied that TRT might have harmful effects on the prognosis of patients with multiple treatment modalities (progression rate: $42.7 \%$ during 15 months of follow-up); however, another database study implied that TRT might be harmless in these populations (rela- tive risk for progression, 1.19; $\mathrm{p}=0.114$; Table 2) [54].

\section{Effects of testosterone replacement therapy in patients with local definitive treatments, according to risk group}

Effects of TRT in patients with local definitive treatments such as radical prostatectomy or radiation therapy were re-assessed according to the disease risk (Table 3). Evidences for effects of TRT in patients with low-risk disease undergoing local treatment comprised 15 studies, which included a total of 315 TRTtreated patients and 89 TRT-untreated controls (Table 3) $[7,33,35,38-41,51-53,55,56,58,59,61]$. All the included 15 studies implied that TRT might be harmless for lowrisk patients (progression rate: $0.0 \%-1.8 \%$ during $6-60$ months of follow-up; Table 3). With regard to patients with intermediate-risk disease, 12 single-armed studies with a total of 126 TRT-treated patients were selected (Table 3) [7,33,35,38-41,51,53,58,59,61]. The results implied that TRT might be harmless for intermediate-risk patients (progression rate: $0.0 \%-7.1 \%$ during $7-60$ months of follow-up; Table 3). On the contrary, with regard to patients with high-risk disease, 11 studies with a total of 85 TRT-treated patients and 15 TRT-untreated controls were selected (Table 3) [33,35,38,40,41,51-53,58,59,61]. Of the 11 studies, the results from 5 studies implied that TRT might be harmful for patients with high-risk disease (progression rate: $18.2 \%-50.0 \%$ during $18-60$ months of follow-up; Table 3). However, as previously mentioned, most of the included studies were single armed, with low quality scores (low-risk, 10; intermediate-risk, 9.5; high-risk, 10).

\section{Effects of testosterone replacement therapy in patients with prostate cancer: results of a meta-analysis}

Of the 36 included studies, four studies were double armed [52,54,56,60]. Underlying prostate cancers were managed with active surveillance in one study [60], radical prostatectomy, in two studies [52,56]; and multiple modalities, in one study [54] (Table 1). Fig. 1 summarizes the result of comparisons between the TRTtreated and TRT-untreated patients. In comparison with TRT-treated and untreated patients, the pooled $\mathrm{RR}$ was not significantly higher than one in comparisons of risk for disease progression (pooled RR, 0.83; 95\% CI, 0.57-1.21; studies, 4). This implies that compared to TRT-untreated patients, TRT-treated patients 


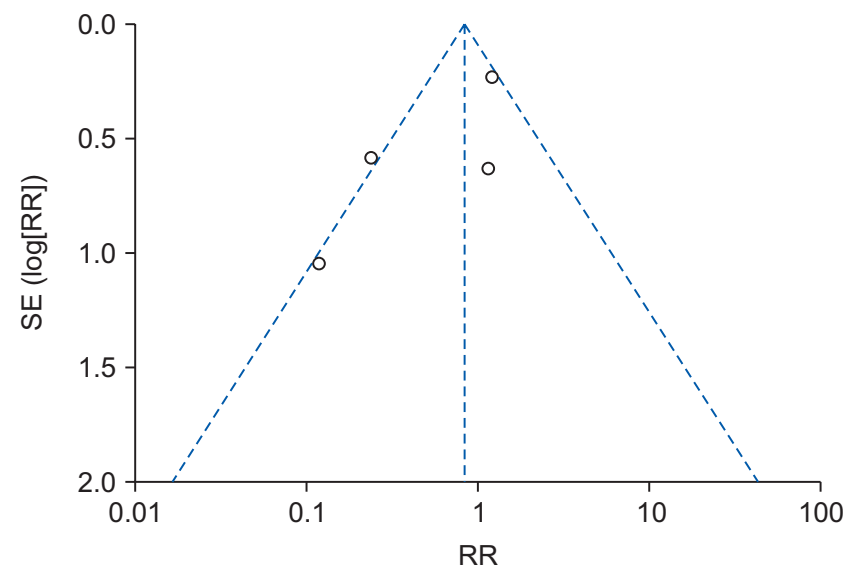

Fig. 3. A funnel graph of the assessment of potential publication bias in studies assessing the risk ratio (RR) for prostate cancer progression in patients with or without testosterone replacement therapy. SE: standard error.

do not have increased risks for disease progression. Despite our attempt to limit inter-study heterogeneity through strict inclusion and exclusion criteria, the heterogeneity between overall treatment outcomes still remained (heterogeneity $\mathrm{X}^{2}$ test, $\mathrm{p}=0.01 ; \mathrm{I}^{2}=72 \%$; Fig. 1 ). However, there was no clear evidence of asymmetry in the funnel plot analysis (Fig. 3). Therefore, it can be concluded that there was no clear evidence of publication bias.

\section{DISCUSSION}

\section{Testosterone replacement therapy and prostate cancer}

Although no randomized controlled trials have been performed to assess TRT and the risk of prostate cancer, evidence to date fails to suggest an increased risk. Calof et al [63] conducted a meta-analysis of 19 placebocontrolled TRT trials and found no significant increase in prostate cancer. A systematic review of 11 placebocontrolled studies by Shabsigh et al [64] showed that men with prostate cancer who received TRT had neither increased risk of prostate cancer nor greater Gleason grade. At a physiologic level, the idea that TRT will not induce development of prostate cancer can be explained by the "saturation model" [65]. As per this theory, while a certain level of testosterone is required to stimulate prostatic growth, higher serum levels do not promote intra-prostatic cancerous growth because of androgen receptor (AR) saturation. This saturation model has been supported by a study that showed that the AR has a maximal binding level for androgen, which occurs at around 60-90 ng/dL [66], and the study demonstrated maintaining a stable intra-prostatic testosterone level irrespective of the levels of circulating testosterone by exogenous testosterone [67].

However, the safety of TRT in patients predisposed to prostate cancer could be a completely different concern. In 1941, Huggins and Hodges [8] already reported that exogenous testosterone stimulates prostate cancer cells, and therefore, leads to disease progression. Similarly, the study by Fowler and Whitmore [32] demonstrated that administration of exogenous testosterone to 52 men with metastatic prostate cancer has been associated with $87 \%$ of unfavorable responses. In contrast, men successfully treated for prostate cancer may not have had any residual cancer cells to be stimulated by androgens. In light of evidence that TRT may not be as harmful to men successfully treated for prostate cancer as once believed, several investigators have reported the use of TRT in men after curative treatment for prostate cancer from the mid-2000s [7,33,35].

\section{Existing evidences for testosterone} replacement therapy in prostate cancer

Our search strategy found 36 eligible studies as existing evidences for TRT in men with untreated and treated prostate cancer (Fig. 2, Table 1). Except for four studies [52,54,56,60], almost studies were single-armed case reports or series with small sample sizes (median number of TRT-treated patients, 20). Therefore, the quality scores measured by the MINOR criteria were also extremely low (median, 9; Table 1). Existing evidences of TRT in men after curative treatment (radical prostatectomy or radiation therapy) for prostate cancer demonstrated relatively good safety outcomes (Table 2). All studies of TRT in patients with radical prostatectomy (studies, 15; TRT-treated, 697 patients; TRT-untreated, 103 patients) implied that TRT might be harmless in patients treated with radical prostatectomy (progression rate: $0.0 \%-6.5 \%$ ). Except for one study (progression rate: 20.0\%) [41], all studies of TRT in patients with radiation therapy (studies, 8; TRTtreated, 270 patients; TRT-untreated, 0 patient) also demonstrated relatively good safety outcomes (progression rate: $0.0 \%-7.7 \%$ ). However, it should be noted that currently available studies are underpowered and their duration is too short to detect any effects attributable to TRT (Table 2). 
Using results from four double-armed studies, data synthesis was performed (Fig. 1). The results of our meta-analysis demonstrated that compared to TRTuntreated patients, TRT-treated patients do not have increased risks for disease progression (pooled RR, 0.83; 95\% CI, 0.57-1.21; Fig. 1). Although our included studies showed no clear evidence of publication bias (Fig. 3), owing to the following limitations of our data, one should be careful when interpreting the results. First, the included studies were heterogeneous. Underlying prostate cancers were managed with active surveillance in one study [60], radical prostatectomy in two studies [52,56], and multiple modalities in one study (Table 1) [54]. Second, the sample size of one database study (TRT-treated, 1,142 patients; TRT-untreated, 148,213 patients) [54] was larger than those of the other three studies [52,56,60]. Therefore, the results of our meta-analysis are likely to converge to the results of a large-scale study (weight, 56.2\%; Fig. 1).

These findings suggest that we do not yet have sufficient evidences for TRT in men with prostate cancer. Therefore, prospective studies are warranted to establish clear evidences for TRT in men with untreated or treated prostate cancer. Currently, an FDA-approved, randomized controlled trial in hypogonadal men is ongoing to investigate the effect of TRT initiated 3 months after radical prostatectomy (Baylor College of Medicine, ClinicalTrials.gov identifier NCT00848497) [68]. The results of these studies are expected to be an important evidence for TRT in men with prostate cancer.

\section{Clinical implications of the current study}

Despite the current study being a small-scale singlearm study with low quality, currently available evidences for TRT in men with prostate cancer suggest the following clinical implications. Firstly, TRT might be harmful in men with advanced disease. In our systematic review, seven of the nine studies demonstrated poor progression rates (38.5\%-100.0\%) following TRT (Table 2). This suggests that exogenous androgens may activate the remaining cancer cells.

Secondly, in men with prostate cancer who undergo active surveillance without definite treatment, caution should be exercised when performing TRT. Despite relatively small-scale underpowered studies (median number of TRT-treated patients, 8), considerable number of studies (2 of 6 studies) have reported high progression rates $(15.4 \%-57.1 \%)$ in men with active surveillance after TRT (Table 2). These results suggest that untreated and remaining prostate cancer cells are likely to be activated and exacerbated by exogenous androgens even if the tumor is at an early stage, with low aggressiveness.

Lastly, even in men in whom prostate cancer has been successfully treated with curative modalities (radical prostatectomy or radiation therapy), attention should be paid to the use of TRT in high-risk disease. While all studies on TRT in men with low-risk (studies, 15; TRT-treated, 315 patients; TRT-untreated, 89 patients) and intermediate-risk disease (studies, 12; TRT-treated, 126 patients; TRT-untreated, 0 patient) implied that TRT might be harmless (progression rate: $0.0 \%-1.8 \%$ and $0.0 \%-7.1 \%$ ), 5 of 11 studies on TRT in men with high-risk disease (TRT-treated, 85 patients; TRT-untreated, 15 patients) revealed relatively high progression rates (18.2\%-50.0\%; Table 3). Even after successful curative treatments (radical prostatectomy or radiation therapy), men with high-risk disease can harbor micrometastases, which cannot be detected by imaging. In these cases, the remaining cancer cells might be affected by exogenous androgens. In summary, TRT may be harmful in men with advanced disease burden, in men with untreated prostate cancer undergoing active surveillance, and in men who have been successfully treated for prostate cancer but had high-risk disease. However, prospective studies are warranted to confirm these hypotheses.

\section{Limitations of the current study}

However, our study has some limitations. First, as previously mentioned, none of the studies included in the current systematic review specified a randomized controlled study design. Therefore, it is difficult to draw any conformational conclusions even after the rigorous reviews. Nevertheless, the present study, which quantitatively assessed the currently available evidences for TRT in men with prostate cancer, is of relatively limited significance. Our result could provide some relevant implications for inadequate TRT candidates in men with prostate cancer. The results of our study can also provide some clues to design further prospective studies. Moreover, owing to the unavailability of sufficient number of studies for data synthesis (4 studies), sensitivity analysis could not be performed. More evidence is required to clarify those 
points. Lastly, in our current meta-analysis, there was tremendous heterogeneity for the included studies (heterogeneity $\mathrm{X}^{2}$ test, $\mathrm{p}=0.01 ; \mathrm{I}^{2}=72 \%$; Fig. 1). Heterogeneity can be caused by numerous factors, such as inclusion criteria, type of treatment, sample size, follow-up period, oncologic outcome parameters, and adjustment for other co-factors. It is also very difficult to explain the inter-study heterogeneity owing to the variability in clinical characteristics across patients within studies. To reduce the heterogeneity-related bias, we adopted the fixed-effects model for data synthesis, which is known to draw more conservative results, and is fit for relatively small number of studies [69]. Despite the limitations, this is the first study to quantitatively analyze the existing evidence for TRT in men with untreated and treated prostate cancer. As a result, some clues about inappropriate patient populations for TRT could be found.

\section{CONCLUSIONS}

Even after the rigorous review, the quality of the currently available evidence was extremely poor. The results of our meta-analysis implied that compared to TRT-untreated patients, TRT-treated patients do not have increased risks for disease progression in prostate cancer. Our systematic review also implied that TRT may be harmful in men with advanced disease burden, in those with untreated prostate cancer undergoing active surveillance, and in those successfully treated for prostate cancer but having high-risk disease. Prospective studies are warranted to confirm these implications.

\section{ACKNOWLEDGEMENTS}

We are indebted to Jung-Yun Lee (E-mail: jungyunlee@yuhs.ac), Assistant Professor, Department of Obstetrics and Gynecology, Yonsei University College of Medicine, for his pro bono technical advice for our systematic review and meta-analysis.

\section{Conflict of Interest}

The authors have nothing to disclose.

\section{Author Contribution}

Conceptualization: MK, SKH. Data curation: MK, SSB. Formal analysis: MK, SSB. Methodology: MK, SSB. Supervision: SKH. Writing - original draft: MK. Writing - review \& editing: SKH.

\section{REFERENCES}

1. Jhaveri FM, Klein EA, Kupelian PA, Zippe C, Levin HS. Declining rates of extracapsular extension after radical prostatectomy: evidence for continued stage migration. J Clin Oncol 1999;17:3167-72.

2. Derweesh IH, Kupelian PA, Zippe C, Levin HS, Brainard J, Magi-Galluzzi C, et al. Continuing trends in pathological stage migration in radical prostatectomy specimens. Urol Oncol 2004;22:300-6.

3. American Cancer Society. Cancer facts \& figures 2016 [Internet]. Atlanta (GA): American Cancer Society; c2016 [cited 2019 Mar 1]. Available from: http://www.cancer.org/research/ cancerfactsstatistics/cancerfactsfigures2016/index.

4. Mottet N, Bellmunt J, Bolla M, Briers E, Cumberbatch MG, De Santis M, et al. EAU-ESTRO-SIOG guidelines on prostate cancer. Part 1: screening, diagnosis, and local treatment with curative intent. Eur Urol 2017;71:618-29.

5. Carroll PH, Mohler JL. NCCN guidelines updates: prostate cancer and prostate cancer early detection. J Natl Compr Canc Netw 2018;16(5S):620-3.

6. Sanda MG, Cadeddu JA, Kirkby E, Chen RC, Crispino T, Fontanarosa J, et al. Clinically localized prostate cancer: AUA/ ASTRO/SUO guideline. Part II: recommended approaches and details of specific care options. J Urol 2018;199:990-7.

7. Kaufman JM, Graydon RJ. Androgen replacement after curative radical prostatectomy for prostate cancer in hypogonadal men. J Urol 2004;172:920-2.

8. Huggins C, Hodges CV. Studies on prostatic cancer. I. The effect of castration, of estrogen and of androgen injection on serum phosphatases in metastatic carcinoma of the prostate. Cancer Res 1941;1:293-7.

9. Morgentaler A. Testosterone therapy for men at risk for or with history of prostate cancer. Curr Treat Options Oncol 2006;7:363-9.

10. Bhasin S, Brito JP, Cunningham GR, Hayes FJ, Hodis HN, Matsumoto AM, et al. Testosterone therapy in men with hypogonadism: an Endocrine Society clinical practice guideline. J Clin Endocrinol Metab 2018;103:1715-44.

11. Wang C, Nieschlag E, Swerdloff R, Behre HM, Hellstrom WJ, Gooren LJ, et al. Investigation, treatment, and monitoring of late-onset hypogonadism in males: ISA, ISSAM, EAU, EAA, 
and ASA recommendations. Eur Urol 2009;55:121-30.

12. Mulhall JP, Trost LW, Brannigan RE, Kurtz EG, Redmon JB, Chiles KA, et al. Evaluation and management of testosterone deficiency: AUA guideline. J Urol 2018;200:423-32.

13. Lizarraga V, Khera M. Contemporary prescribing patterns of testosterone replacement therapy in hypogonadal patients with a history of prostate cancer. J Urol 2015;193(4S):e625.

14. Millar AC, Elterman DS, Goldenberg L, Van Asseldonk B, Curtis A, Jarvi K. A survey of Canadian urologists' opinions and prescribing patterns of testosterone replacement therapy in men on active surveillance for low-risk prostate cancer. Can Urol Assoc J 2016;10:181-4.

15. Dorff TB, Vogelzang NJ. Use of testosterone replacement therapy in patients with prostate cancer. Curr Urol Rep 2011;12:223-8.

16. Gravina GL, Di Sante S, Limoncin E, Mollaioli D, Ciocca G, Carosa E, et al. Challenges to treat hypogonadism in prostate cancer patients: implications for endocrinologists, urologists and radiotherapists. Transl Androl Urol 2015;4:139-47.

17. Kaplan AL, Hu JC, Morgentaler A, Mulhall JP, Schulman CC, Montorsi F. Testosterone therapy in men with prostate cancer. Eur Urol 2016;69:894-903.

18. Kaufman J. A rational approach to androgen therapy for hypogonadal men with prostate cancer. Int J Impot Res 2006;18:26-31.

19. Khera M, Crawford D, Morales A, Salonia A, Morgentaler A. A new era of testosterone and prostate cancer: from physiology to clinical implications. Eur Urol 2014;65:115-23.

20. Morgentaler A. Testosterone therapy in men with prostate cancer: scientific and ethical considerations. J Urol 2009;181:972-9.

21. Pastuszak AW, Rodriguez KM, Nguyen TM, Khera M. Testosterone therapy and prostate cancer. Transl Androl Urol 2016;5:909-20.

22. Stroup DF, Berlin JA, Morton SC, Olkin I, Williamson GD, Rennie D, et al. Meta-analysis of observational studies in epidemiology: a proposal for reporting. Meta-analysis $\mathrm{Of} \mathrm{Ob}-$ servational Studies in Epidemiology (MOOSE) group. JAMA 2000;283:2008-12.

23. Moher D, Liberati A, Tetzlaff J, Altman DG. Preferred reporting items for systematic reviews and meta-analyses: the PRISMA statement. Ann Intern Med 2009;151:264-9, W64.

24. Slim K, Nini E, Forestier D, Kwiatkowski F, Panis Y, Chipponi J. Methodological index for non-randomized studies (MINORS): development and validation of a new instrument. ANZ J Surg 2003;73:712-6.

25. Mantel N. Chi-square tests with one degree of freedom; extensions of the Mantel-Haenszel Procedure. J Am Stat Assoc
1963;58:690-700.

26. Higgins JP, Thompson SG, Deeks JJ, Altman DG. Measuring inconsistency in meta-analyses. BMJ 2003;327:557-60.

27. DerSimonian R, Laird N. Meta-analysis in clinical trials revisited. Contemp Clin Trials 2015;45(Pt A):139-45.

28. Harbord RM, Egger M, Sterne JA. A modified test for smallstudy effects in meta-analyses of controlled trials with binary endpoints. Stat Med 2006;25:3443-57.

29. Pearson OH. Discussion of Dr. Huggins' paper: control of cancers of man by endocrinological methods. Cancer Res 1957;17:473-9.

30. Prout GR Jr, Brewer WR. Response of men with advanced prostatic carcinoma to exogenous administration of testosterone. Cancer 1967;20:1871-8.

31. Morales A, Connolly JG, Bruce AW. Androgen therapy in advanced carcinoma of the prostate. Can Med Assoc J 1971;105:71-2.

32. Fowler JE Jr, Whitmore WF Jr. The response of metastatic adenocarcinoma of the prostate to exogenous testosterone. J Urol 1981;126:372-5.

33. Agarwal PK, Oefelein MG. Testosterone replacement therapy after primary treatment for prostate cancer. J Urol 2005;173:533-6.

34. Ferreira U, Leitao VA, Denardi F, Matheus WE, Stopiglia RM, Netto NR Jr. Intermittent androgen replacement for intense hypogonadism symptoms in castrated patients. Prostate Cancer Prostatic Dis 2006;9:39-41.

35. Sarosdy MF. Testosterone replacement for hypogonadism after treatment of early prostate cancer with brachytherapy. Cancer 2007;109:536-41.

36. Davila $\mathrm{HH}$, Arison CN, Hall MK, Salup R, Lockhart JM, Carrion RE. Analysis of the PSA response after testosterone supplementation in patients who have previously received management for their localized prostate cancer. J Urol 2008;179:428.

37. Mathew P. Prolonged control of progressive castrationresistant metastatic prostate cancer with testosterone replacement therapy: the case for a prospective trial. Ann Oncol 2008;19:395-6.

38. Nabulsi O, Tal R, Gotto G, Narus J, Goldenberg L, Mulhall JP. Outcomes analysis of testosterone supplementation in hypogonadal men following radical prostatectomy. J Urol 2008;179(4S):426-7.

39. Pushkar DY, Govorov AV, Segal AS. Androgen replacement therapy in patients with age-related hypogonadism after radical retropubic prostatectomy for localized prostate cancer. J Mens Health 2008;5:234-8

40. Khera M, Grober ED, Najari B, Colen JS, Mohamed O, Lamb 
DJ, et al. Testosterone replacement therapy following radical prostatectomy. J Sex Med 2009;6:1165-70.

41. Morales A, Black AM, Emerson LE. Testosterone administration to men with testosterone deficiency syndrome after external beam radiotherapy for localized prostate cancer: preliminary observations. BJU Int 2009;103:62-4.

42. Morgentaler A. Two years of testosterone therapy associated with decline in prostate-specific antigen in a man with untreated prostate cancer. J Sex Med 2009;6:574-7.

43. Morris MJ, Huang D, Kelly WK, Slovin SF, Stephenson RD, Eicher $\mathrm{C}$, et al. Phase 1 trial of high-dose exogenous testosterone in patients with castration-resistant metastatic prostate cancer. Eur Urol 2009;56:237-44.

44. Szmulewitz R, Mohile S, Posadas E, Kunnavakkam R, Karrison T, Manchen E, et al. A randomized phase 1 study of testosterone replacement for patients with low-risk castrationresistant prostate cancer. Eur Urol 2009;56:97-103.

45. Isbarn H, Fisch M, Huland H, Graefen M, Sommer F. Testosterone treatment after radical prostatectomy for pathologically organ-confined prostate cancer; results from a multiinstitutional study. J Urol 2010;183(4S):e576-e7.

46. Leibowitz RL, Dorff TB, Tucker S, Symanowski J, Vogelzang NJ. Testosterone replacement in prostate cancer survivors with hypogonadal symptoms. BJU Int 2010;105:1397-401.

47. Sathyamoorthy K, Mohamed O, Stein M, Lipshultz L, Khera M. Follow-up series of testosterone replacement therapy following radical prostatectomy: the Baylor experience. J Sex Med 2010;7(Suppl 1):10-1.

48. Morales A. Effect of testosterone administration to men with prostate cancer is unpredictable: a word of caution and suggestions for a registry. BJU Int 2011;107:1369-73.

49. Morgentaler A, Lipshultz LI, Bennett R, Sweeney M, Avila D Jr, Khera M. Testosterone therapy in men with untreated prostate cancer. J Urol 2011;185:1256-60.

50. Matsushita K, Katz D, Stember D, Nelson C, Mulhall J. Analysis of the safety and efficacy of testosterone suplementation following radical prostatectomy. J Sex Med 2012;9(Suppl 4):205.

51. Pastuszak AW, Pearlman AM, Godoy G, Miles BJ, Lipshultz LI, Khera M. Testosterone replacement therapy in the setting of prostate cancer treated with radiation. Int J Impot Res 2013;25:24-8.

52. Pastuszak AW, Pearlman AM, Lai WS, Godoy G, Sathyamoorthy K, Liu JS, et al. Testosterone replacement therapy in patients with prostate cancer after radical prostatectomy. J Urol 2013;190:639-44.

53. Balbontin FG, Moreno SA, Bley E, Chacon R, Silva A, Morgentaler A. Long-acting testosterone injections for treatment of testosterone deficiency after brachytherapy for prostate cancer. BJU Int 2014;114:125-30.

54. Kaplan AL, Trinh QD, Sun M, Carter SC, Nguyen PL, Shih YT, et al. Testosterone replacement therapy following the diagnosis of prostate cancer: outcomes and utilization trends. J Sex Med 2014;11:1063-70.

55. Nakano K, Kiuchi H, Miyagawa Y, Tsujimura A, Nonomura $\mathrm{N}$. [Testosterone replacement therapy for late-onset hypogonadism after radical prostatectomy: a case report]. Hinyokika Kiyo 2014;60:397-400. Japanese.

56. Wynia B, Lee T, Taneja S, Lepor H, Huang W, Stifelman M, et al. Testosterone replacement therapy in patients with prostate cancer after prostatectomy: a 5-year single center experience. J Urol 2014;191(4S):e527-8.

57. Berookhim B, Krishnan R, Nelson C, Mulhall JP. Testosterone replacement therapy in men undergoing active surveillance for prostate cancer. J Sex Med 2015;12(Suppl 1):13.

58. Kühn CM, Strasser H, Romming A, Wullich B, Goebell PJ. Testosterone replacement therapy in hypogonadal men following prostate cancer treatment: a questionnaire-based retrospective study among urologists in Bavaria, Germany. Urol Int 2015;95:153-9.

59. Pastuszak AW, Khanna A, Badhiwala N, Morgentaler A, Hult $\mathrm{M}$, Conners WP, et al. Testosterone therapy after radiation therapy for low, intermediate and high risk prostate cancer. J Urol 2015;194:1271-6.

60. Kacker R, Hult M, San Francisco IF, Conners WP, Rojas PA, Dewolf WC, et al. Can testosterone therapy be offered to men on active surveillance for prostate cancer? Preliminary results. Asian J Androl 2016;18:16-20.

61. Ory J, Flannigan R, Lundeen C, Huang JG, Pommerville P, Goldenberg SL. Testosterone therapy in patients with treated and untreated prostate cancer: impact on oncologic outcomes. J Urol 2016;196:1082-9.

62. Morgentaler A, Magauran D, Neel D, Krakowsky Y, Conners W. Recurrence rates following testosterone therapy in a large clinical cohort of men with prostate cancer. J Urol 2018;199(4S):e206.

63. Calof OM, Singh AB, Lee ML, Kenny AM, Urban RJ, Tenover $\mathrm{JL}$, et al. Adverse events associated with testosterone replacement in middle-aged and older men: a meta-analysis of randomized, placebo-controlled trials. J Gerontol A Biol Sci Med Sci 2005;60:1451-7.

64. Shabsigh R, Crawford ED, Nehra A, Slawin KM. Testosterone therapy in hypogonadal men and potential prostate cancer risk: a systematic review. Int J Impot Res 2009;21:9-23.

65. Morgentaler A. Testosterone replacement therapy and prostate cancer. Urol Clin North Am 2007;34:555-63, vii. 
66. Traish AM, Williams DF, Hoffman ND, Wotiz HH. Validation of the exchange assay for the measurement of androgen receptors in human and dog prostates. Prog Clin Biol Res 1988;262:145-60.

67. Marks LS, Hess DL, Dorey FJ, Macairan ML. Prostatic tissue testosterone and dihydrotestosterone in African-American and white men. Urology 2006;68:337-41.

68. Testosterone for penile rehabilitation after radical prostatec- tomy [Internet]. Bethesda (MD): U.S. National Library of Medicine; c2015 [cited 2019 Mar 1]. Available from: https:// clinicaltrials.gov/ct2/show/NCT00848497.

69. Kim SY, Park JE, Seo HJ, Lee YJ, Jang BH, Son HJ, et al. NECA's guideline for undertaking systemic reviews and metaanalyses for intervention. Seoul, Korea: National Evidencebased Healthcare Collaborating Agency (NECA); 2011. 
Appendix. Detailed query settings for search strategy

\begin{tabular}{|c|c|}
\hline Search & Query \\
\hline$\# 1$ & Search "Prostatic Neoplasms"[MeSH] \\
\hline$\# 2$ & Search prostat ${ }^{*}$ cancer \\
\hline$\# 3$ & Search prostat* carcinoma \\
\hline$\# 4$ & Search prostat* adenocarcinoma \\
\hline$\# 5$ & Search (\#1 or \#2 or \#3 or \#4) \\
\hline \#6 & Search "Hormone Replacement Therapy"[MeSH] \\
\hline$\# 7$ & Search testosterone replacement \\
\hline$\# 8$ & Search testosterone therapy \\
\hline$\# 9$ & Search testosterone supplementation \\
\hline$\# 10$ & Search androgen replacement \\
\hline$\# 11$ & Search androgen therapy \\
\hline$\# 12$ & Search androgen supplementation \\
\hline$\# 13$ & Search (\#6 or \#7 or \#8 or \#9 or \#10 or \#11 or \#12) \\
\hline \#14 & Search (\#5 and \#13) \\
\hline$\# 15$ & Search "Hypogonadism"[MeSH] \\
\hline$\# 16$ & Search testosterone deficiency \\
\hline$\# 17$ & Search androgen deficiency \\
\hline$\# 18$ & Search (\#15 or \#16 or \#17) \\
\hline$\# 19$ & Search (\#14 and \#18) \\
\hline \#20 & Search "Watchful Waiting"[MeSH] \\
\hline \#21 & Search surveillance \\
\hline \#22 & Search "High-Intensity Focused Ultrasound Ablation"[MeSH] \\
\hline \#23 & Search HIFU \\
\hline$\# 24$ & Search "Cryotherapy"[MeSH] \\
\hline \#25 & Search cryo* \\
\hline \#26 & Search focal therapy \\
\hline \#27 & Search focal treatment \\
\hline \#28 & Search "Prostatectomy"[MeSH] \\
\hline \#29 & Search prostatectomy \\
\hline \#30 & Search "Radiotherapy"[MeSH] \\
\hline$\# 31$ & Search radiation \\
\hline$\# 32$ & Search "Brachytherapy"[MeSH] \\
\hline \#33 & Search brachy* \\
\hline \#34 & Search "Drug Therapy"[MeSH] \\
\hline \#35 & Search chemo* \\
\hline \#36 & Search androgen deprivation \\
\hline \#37 & Search hormone* \\
\hline \#38 & Search (\#20 or \#21 or \#22 or \#23 or \#24 or \#25 or \#26 or \#27 or \#28 or \#29 or \#30 or \#31 or \#32 or \#33 or \#34 or \#35 or \#36 or \#37) \\
\hline \#39 & Search (\#19 and \#38) \\
\hline
\end{tabular}

Presented as query form of PubMed.

Core logics of search queries were not different in other database searches. 